\title{
CÁNCER EN MUJERES EMBARAZADAS Y SU EXTENSIÓN A LA UNIDAD FETOPLACENTARIA
}

\section{Cancer in pregnant women and its extension to fetoplacental unit}

\author{
Eugenia Corredor-Sánchez, M.D.*, Marcos Castillo M.D.**
}

Recibido: octubre 18/06 - Revisado: abril 18/07 - Aceptado: septiembre 13/07

\section{RESUIMEN}

Debido a la rara presentación simultánea entre cáncer y embarazo y a que la afectación de la unidad fetoplacentaria por dicha patología es aún más exótica, la presente revisión busca lograr un mejor entendimiento sobre la afectación placentaria y fetal por patologías malignas que pueden comprometer el embarazo. Se realizó una búsqueda en diferentes bases de datos electrónicas como Pubmed, Ovid, Elsevier, Cochrane y libros de texto impresos. Los hallazgos en la literatura médica son bastante limitados y básicamente se trata de reportes de casos o series de casos, encontrados mediante búsqueda bibliográfica.

Desde el primer reporte de metástasis de carcinoma hepático a placenta y feto, descrito en 1866, se han documentado solamente 14 casos de metástasis transplacentaria materno-fetal y poco más de 50 casos de enfermedades malignas maternas con metástasis a placenta.

Palabras clave: metástasis, fetoplacentaria, embarazo, cáncer, transmisión vertical.

\section{SUIMMARY}

Due to the rare simultaneous presentation of cancer during pregnancy and a fetoplacental unit being affected by such pathology is even

* Ginecóloga y obstetra. Departamento de Ginecología y Obstetricia. Hospital Universitario de la Samaritana.

Carrera 8 Número 0-55 Sur. Bogotá, Colombia. Universidad Javeriana.

Correo electrónico: pilitaney@hotmail.com

** Ginecólogo y obstetra. Instructor Universidad de la Sabana. rarer then this present review sought for better understanding regarding placental and fetal units being affected by malign pathologies which could compromise pregnancy. Different databases were searched (i.e. Pubmed, Ovid, Elsevier and Cochrane) as were medical texts and books. Findings in the medical literature were extremely limited, basically dealing with case-reports and case series found by a bibliographic search.

Only 14 cases of maternal-fetal trans-placenta metastasis have been documented since the first report of hepatic carcinoma metastasis of the placenta and fetus described in 1866 and only just over 50 cases of malign maternal diseases involving metastasis of the placenta.

Key words: metastasis, fetoplacental unit, pregnancy, cancer, vertical transmission.

\section{INTRODUCCIÓN}

La complicación del embarazo por el cáncer es de rara coexistencia. Los tumores malignos en mujeres embarazadas tienen diferentes orígenes e involucran: cáncer de seno, cáncer cervical, enfermedad de Hodgkin, melanoma maligno, leucemia, cáncer de ovario y cáncer intestinal. No se ha visto que el embarazo tenga influencia adversa en tales patologías malignas.

La transmisión vertical del cáncer es extremadamente rara y poco probable debido a la barrera placentaria y el sistema inmune fetal. Es de esperar que por su gran flujo sanguíneo, área de superficie 
y ambiente biológico favorable para el crecimiento, la placenta sea un sitio ideal para metástasis, pero hay factores que juegan un papel modulador en la transmisión transplacentaria de malignidad.

La presente revisión busca lograr un mejor entendimiento sobre la afectación placentaria y fetal por patologías malignas que pueden comprometer el embarazo. Se realizó una búsqueda en diferentes bases de datos electrónicas como Pubmed, Ovid, Elsevier, Cochrane y libros de texto impresos. Los hallazgos en la literatura médica son bastante limitados y básicamente se trata de reportes de casos o series de casos, encontrados mediante búsqueda bibliográfica.

\section{Epidemiología}

La complicación del embarazo por el cáncer es de rara coexistencia. La incidencia es aproximadamente 1 en 1.000 embarazos.

Mientras que los tumores más comunes en la edad reproductiva de la mujer (15 a 44 años) son: carcinoma de tiroides, carcinoma cervical, melanoma y linfoma; las neoplasias malignas reportadas en mujeres embarazadas tienden a tener diferentes orígenes e involucran: cáncer de seno, cáncer cervical, enfermedad de Hodgkin, melanoma maligno, leucemia, cáncer de ovario y cáncer intestinal. ${ }^{1}$

La mayoría de tipos de cáncer maternos diagnosticados durante el embarazo tienen resultados favorables para la madre y el feto, y no se ha visto que el embarazo muestre una influencia adversa en el marco de estas patologías malignas. ${ }^{2}$

La transmisión vertical del cáncer es extremadamente rara, aunque las células maternas alcancen al feto. El primer reporte de metástasis a la placenta y el feto fue descrito en 1866. En este caso Friederich observó una madre con carcinoma hepático diseminado que se propagó al feto causándole la muerte.

Posteriormente se han reportado más de 50 casos de enfermedades malignas maternas con metástasis a la placenta y 14 casos documentados de metástasis transplacentaria materno-fetal en los últimos 137 años. $^{2}$

\section{Biología de la transmisión del cáncer}

La transmisión del cáncer de la madre al feto es poco probable debido a la barrera placentaria y al sistema inmune fetal. Por tanto hay una discrepancia entre la incidencia de cáncer durante el embarazo y los casos de compromiso fetal o placentario.

Es de esperar que por su generoso flujo sanguíneo, su gran área de superficie y el ambiente biológico favorable para el crecimiento, la placenta sea un sitio ideal para metástasis, por tanto la relativa escasez de tal evento es poco clara. ${ }^{3}$

Hay varios factores que pueden jugar un papel en la modulación de la transmisión transplacentaria de malignidad:

1. Casi todos los casos de compromiso fetoplacentario por cáncer materno han ocurrido en mujeres embarazadas con diseminación extensa de la enfermedad, lo que favorece la hipótesis de metástasis hematógenas arteriales, en oposición a la diseminación por vía linfática, drenaje venoso o por continuidad.

2. Una vez que las células tumorales son atrapadas en el espacio intervelloso, su entrada puede ser impedida al lado fetal, por la barrera trofoblástica placentaria.

3. El sistema inmune fetal puede jugar un papel en el rechazo de las células tumorales, visto como un homoinjerto por el feto. ${ }^{4}$

La fisiología y la inmunología de los casos raros de metástasis feto-placentaria ha generado hipótesis en cuanto a la generación de enfermedad maligna en el feto.

Como el flujo sanguíneo útero-placentario representa el 10\% del rendimiento cardíaco materno, se esperaría la diseminación hematógena de émbolos tumorales maternos a la placenta. Sin embargo, la circulación materna y fetal están separadas por trofoblasto, vellosidades coriónicas y endotelio capilar; esta barrera placentaria, en combinación con el sistema inmune fetal, es marcadamente eficiente en la prevención de la diseminación de aloinjertos de tumor materno al feto. 
Pero, a pesar de la naturaleza protectora, la transmisión al feto de células maternas neoplásicas y no neoplásicas ocurre durante el embarazo, porque si se altera la separación del sistema sanguíneo materno y fetal, las células tumorales intravasculares maternas pueden pasar a través de la placenta y terminar en el hígado fetal por vía de la vena umbilical o en el pulmón fetal por vía del ductus venoso.

Los fetos con inmunodeficiencia congénita están probablemente en más alto riesgo para el injerto de células tumorales. Otros factores que pueden afectar la probabilidad de la entrada de células tumorales a la circulación fetal, incluyen:

1. La homocigocidad materna para uno de los haplotipos de HLA fetal.

2. El potencial metastásico del tumor materno.

3. Alta carga sanguínea tumoral materna y/o placentaria. ${ }^{1,4}$

En todos los casos de compromiso de metástasis a la unidad feto-placentaria se encontró engrosamiento macroscópico de la placenta y microscópicamente se hallaron células tumorales en el espacio intervelloso, con hallazgos morfológicos característicos de células malignas, tales como: variación del tamaño, notable pleomorfismo nuclear con células binucleadas, citoplasma abundante con algunas vacuolas diminutas. ${ }^{5}$

\section{Distribución del compromiso metastásico fetoplacentario}

La metástasis transplacentaria y el injerto de células tumorales maternas son raros, con un total de 14 casos reportados desde 1866. Estos casos incluyen: 5 melanomas, 3 linfomas, 3 leucemias, 3 carcinomas.

Debido al retraso entre el tiempo del nacimiento y el diagnóstico, en muchos de estos niños, los casos de transmisión materno-fetal pueden no ser tan raros como lo sugiere la literatura y el número de casos podría ser más alto. ${ }^{2}$

Los tipos de cáncer que se han reportado hacen metástasis al espacio intervelloso o a la placenta, sin compromiso de metástasis al feto, incluyen en orden de frecuencia: melanomas, carcinomas, especialmente cáncer de seno, linfomas, leucemias y sarcomas. ${ }^{6}$ Contrario al pronóstico desfavorable para mujeres con metástasis placentaria, el pronóstico para tales infantes ha sido excelente.

Se ha reportado también el caso de una mujer con embarazo de 33 semanas y un tumor neuroectodérmico primitivo en el retroperitoneo, confirmado histológicamente, sin evidencia de compromiso del neonato nacido por cesárea, pero con examen microscópico placentario que reveló infiltración de células tumorales al espacio intervelloso. ${ }^{7}$

\section{Transmisión vertical del cáncer}

\section{a. Melanoma}

El melanoma constituye únicamente el $8 \%$ de los tumores malignos que ocurren durante el embarazo; este es el tipo de cáncer más común que hace metástasis a la placenta y al feto. La revisión más extensa muestra que el melanoma constituye aproximadamente el 30\% de todos los casos reportados de tumores maternos que hacen metástasis a la placenta. ${ }^{6}$ Aproximadamente el 58\% de los casos que involucran al feto, correspondieron a melanomas. ${ }^{8}$

Es importante notificar al patólogo, la posibilidad de metástasis placentaria para todas las pacientes con melanoma invasivo, ya que dicha metástasis es indicativa de enfermedad en estadio IV y puede guiar el tratamiento apropiado para la madre. Así mismo, el conocimiento de que una placenta está libre de enfermedad metastásica, puede ofrecer tranquilidad a los padres, del bajo riesgo de metástasis al feto. ${ }^{8}$

Ningún caso de desarrollo de metástasis después de los 11 meses de edad ha sido reportado; sin embargo es teóricamente posible que puedan ocurrir metástasis tardías en niños y en adultos. ${ }^{8}$

En los niños que desarrollan metástasis fetal de melanoma materno, son más frecuentes como manifestaciones iniciales, las lesiones en piel y edema abdominal; posterior a la aparición de las mismas, se ha descrito compromiso hepático y la muerte 
También se ha reportado un caso de muerte fetal intrauterina. ${ }^{9}$

Está descrito que la frecuencia de metástasis fetales de melanoma diagnosticado durante el embarazo, es menor del $1 \% .^{10}$

La prematuridad fue una complicación común de los niños nacidos con metástasis placentaria de melanoma, con una edad gestacional media de 34 semanas, pero la tasa de mortalidad secundaria a la misma fue baja. ${ }^{9}$

Ha sido reportado en la literatura un caso de melanoma ocular coroidal primario metastásico a hígado, esqueleto y placenta, cuyo examen macroscópico no mostró evidencia de enfermedad metastásica; sin embargo, al examen microscópico hubo evidencia de células tumorales en el espacio intervelloso, con contenido de pigmento café-negruzco y positivo con S-100 y HMB-45, compatible con melanoma de enfermedad metastásica a la placenta.

La valoración del feto por examen físico y la ultrasonografía abdominal no detectaron evidencia de compromiso fetal. ${ }^{11}$

Se cree que el melanoma coroidal ocular es un tumor de alto riesgo con potencial significativo para diseminación sistémica subsecuente, particularmente a hígado y a la unidad feto-placentaria cuando la mujer queda en embarazo; por esto, todas las mujeres con historia de este tumor deberían ser informadas del riesgo potencial descrito, cuando deseen quedar en embarazo. ${ }^{11}$

\section{b. Linfoma y leucemia}

La incidencia de leucemia aguda durante el embarazo se estima que es similar a la población general. La incidencia de enfermedad de Hodgkin se considera que es de 1:1.000 a 1:6.000 embarazos, mientras que la del linfoma no Hodgkin es más rara. ${ }^{2}$

El linfoma no Hodgkin ha sido asociado durante el embarazo con alto grado histológico, enfermedad diseminada y alta mortalidad materna.

Algunos investigadores han reportado que no existe diferencia clínica en el comportamiento del linfoma durante el embarazo, mientras que otros han considerado un efecto protector del embarazo sobre el curso del linfoma. ${ }^{5}$

En 1986 se documentó por primera vez compromiso placentario por linfoma no Hodgkin materno. En este y en los demás casos reportados, el compromiso de la placenta fue macroscópicamente visible y las células tumorales microscópicas se encontraron en el espacio intervelloso.

Han sido reportados seis tumores hematológicos con transmisión materno-fetal: 3 linfomas (1 linfosarcoma, 1 enfermedad de Hodgkin y 1 linfoma de células asesinas naturales), 1 caso de leucemia mieloide aguda y 2 casos de leucemia linfoide aguda.

\section{c. Carcinoma}

Tres de los catorce tumores metastásicos transplacentarios fueron carcinomas. En este grupo se incluye el primer caso reportado en 1866, que involucró la diseminación de un carcinoma hepático, reconocido al nacimiento.

El segundo caso fue un adenocarcinoma materno de pulmón, que se presentó como una metástasis a los dos meses de edad; después de una escisión local de la metástasis, el niño fue reportado libre de enfermedad hasta cinco años después del diagnóstico.

El tercer caso de este grupo es de un carcinoma pulmonar de célula pequeña con metástasis al feto, con madre con antecedente de tabaquismo. El compromiso de la placenta fue evidente en el momento del parto; el niño estuvo bien hasta la edad de cinco meses, cuando nódulos en hígado y pulmón fueron notados por TAC.

El análisis histológico e inmunohistoquímico de la madre, la placenta y las biopsias de hígado del infante fueron similares y consistentes con transmisión transplacentaria de carcinoma de célula pequeña del pulmón.

El infante fue tratado con quimioterapia seguida por transplante autólogo de médula ósea, después tuvo una recaída a nivel del sistema nervioso central, 
la metástasis fue resecada y se trató con radioterapia y quimioterapia adicional; sin embargo la enfermedad nuevamente recurrió y el infante murió a los 23 meses de edad. ${ }^{12}$

\section{Evaluación del recién nacido de la mujer con cáncer}

Las guías para la evaluación de los niños nacidos de mujeres con cáncer, son escasas en la literatura. Se recomienda que un niño nacido de una mujer con enfermedad maligna activa o en sospecha debe inicialmente tener un examen físico completo, conteo sanguíneo, evaluación metabólica, pruebas de función hepática, de coagulación, lactato deshidrogenasa sérica, nivel de ácido úrico, uroanálisis, RNM y TAC de tórax, abdomen y pelvis.

Adicionalmente la placenta deber ser examinada macroscópica y microscópicamente para involucrar el tumor.

Casos de metástasis materno-fetal se han presentado hasta varios meses después del nacimiento, por lo tanto la vigilancia continua debe ser establecida durante el primer año de vida del niño.

\section{REFERENCIAS}

1. Tolar J, Neglia JP. Transplacental and other routes of cancer transmission between individuals. J Pediatr Hematol Oncol 2003;25:430-4.

2. Pavlidis NA. Coexistence of pregnancy and malignancy. Oncologist 2002;7:279-87.

3. Creasy RK, Resnik R. Pelvic malignancies, gestational trophoblastic neoplasia and nonpelvic malignancies. En: Creasy RK, Resnik R, eds. Maternal-fetal medicine. 5th edition. Philadelphia: Saunders; 2004; Chapter 58. p. 1213-42.

4. Brossard J, Abish S, Bernstein ML, Baruchel S, Kovacs L, Pollack R. Maternal malignancy involving the products of conception: a report of malignant melanoma and medulloblastoma. Am J Pediatr Hematol Oncol 1994;16:380-3.

5. Meguerian-Bedoyan Z, Lamant L, Hopfner C, Pulford K, Chittal S, Delsol G. Anaplastic large cell lymphoma of maternal origin involving the placenta: case report and literature survey. Am J Surg Pathol 1997;21: 1236-41.

6. Salamon MA, Sherer DM, Saller DN Jr, Metlay LA, Sickel JZ. Placental metastases in a patient with recurrent breast carcinoma. Am J Obstet Gynecol 1994;171:573-4.

7. Sakurai H, Mitsuhashi N, Ibuki Y, Joshita T, Fukusato T, Niibe H. Placental metastasis from maternal primitive neuroectodermal tumor. Am J Clinic Oncol 1998;21:39-41.

8. Altman JF, Lowe L, Redman B, Esper P, Schwartz JL, Johnson TM, et al. Placental metastasis of maternal melanoma. J Am Acad Dermatol 2003; 49:1150-4.

9. Alexander A, Samlowski WE, Grossman D, Bruggers CS, Harris RM, Zone JJ, et al. Metastic melanoma in pregnancy: risk of transplacental metastases in the infant. J Clinic Oncol 2003;21:2179-86.

10. Borden EC. Melanoma and pregnancy. Semin Oncol 2000;27:654-6.

11. Marsh RD, Chu NM. Placental metastasis from primary ocular melanoma: a case report. Am J Obstet Gynecol 1996;174:1654-5.

12. Tolar J, Coad JE, Neglia JP. Transplacental transfer of small-cell carcinoma of the lung. $N$ Engl J Med 2002;346:1501-2. 